\title{
Randomized comparison of phone versus in-person BRCA1/2 predisposition genetic test result disclosure counseling
}

Jean Jenkins, PhD, RN, FAAN ${ }^{1}$, Kathleen A. Calzone, MSN, RN, APNG, FAAN ${ }^{2}$, Eileen Dimond, MS, RN ${ }^{2}$, David J. Liewehr, $M S^{3}$, Seth M. Steinberg, PhD $D^{3}$, Oxana Jourkiv, $M D^{2}$, Pam Klein, $M D^{4}$, Peter W. Soballe, $M D^{5}$, Sheila A. Prindiville, $M D, M P H^{2}$, and Ilan R. Kirsch, $M D^{6}$

\begin{abstract}
Purpose: This study evaluated whether phone results were equivalent to in-person result disclosure for individuals undergoing BRCA1/2 predisposition genetic testing. Methods: A total of 111 of 136 subjects undergoing education and counseling for $B R C A 1 / 2$ predisposition genetic testing agreed to randomization to phone or in-person result disclosure. Content and format for both sessions were standardized. Data from the State-Trait Anxiety Inventory and the Psychological General Well-Being index were collected at baseline and then again at 1 week and 3 months after disclosure of test results. Baseline measures were administered after the following had occurred: counseling/education session had been conducted, informed consent had been obtained, and decision to be tested had been made. Satisfaction and cost assessments were administered after the result session. At 1 week, participants were asked their preferred method of result disclosure. Results: There were no differences in anxiety and general well-being measures between 50 phone and 52 in-person results disclosure. Both groups reported similar rates of satisfaction with services. Among those with a preference, $77 \%$ preferred the notification method assigned. There was a statistically significant preference for phone results among the $23 \%$ who did not prefer the method assigned. Greater costs were associated with in-person result disclosure. Conclusions: These data suggest that phone results are a reasonable alternative to traditional in-person BRCA1/2 genetic test disclosure without any negative psychologic outcomes or compromise in knowledge. However, further study is needed in a more clinically representative population to confirm these findings. Genet Med 2007:9(8):487-495.
\end{abstract}

Key Words: genetic testing, genetic counseling, BRCA1/BRCA2, result disclosure, risk communication

Breast cancer is one of the most common forms of cancer in American women and the second leading cause of cancer mortality. More than 214,000 new breast cancer cases will be diag-

From the ${ }^{1}$ National Institutes of Health, National Human Genome Research, ${ }^{2}$ National Cancer Institute, Center for Cancer Research, Genetics Branch, and ${ }^{3}$ National Cancer Institute, Center for Cancer Research, Biostatistics and Data Management Section, Bethesda, Maryland; ${ }^{4}$ Genentech, Inc., San Francisco, California; ${ }^{5}$ Uniformed Services University, Bethesda, Maryland; and ${ }^{6}$ Amgen, Inc., Seattle, Washington.

Disclosure: Dr. Kirsch is currently employed by Amgen Inc. Dr. Klein is currently employed by Genentech, Inc. As such they receive salary and stock options from these companies. They accepted their positions after the work described in the article (Jenkins et. al.) had been completed. Furthermore, there is no aspect of this paper that impacts the current scope of their professional activities which, at this time, are focused on drug development as opposed to genetic counseling. No other authors declare any conflict of interest.

Kathleen A. Calzone, RN, MSN, APNG, FAAN, National Cancer Institute, Center for Cancer Research-Genetics Branch, 8901 Wisconsin Ave., Bldg 8 Room 5101, Bethesda, MD 208895105.E-Mail: calzonek@mail.nih.gov.

Submitted for publication February 7, 2007.

Accepted for publication May 2, 2007.

Supported by Intramural Research Program of the National Institutes of Health, National Cancer Institute, Center for Cancer Research.

The Chief, Navy Bureau of Medicine and Surgery, Washington, DC, Clinical Investigation Program No. B99-015. nosed in 2006, and approximately $5 \%$ to $10 \%$ of those cases will be associated with an inherited germline mutation in a dominant susceptibility allele. ${ }^{1,2}$ As a consequence, the absolute number of breast cancer cases diagnosed annually that are associated with a genetic predisposition is considerable. The most common high penetrance breast cancer susceptibility alleles are $B R C A 1$ and $B R C A 2$, which account for at least half of all genetically predisposed breast cancer. ${ }^{3-5}$ Family history has long been recognized as a risk factor for breast cancer, but it was not until the availability of clinical testing for mutations in $B R C A 1$ and BRCA2 that breast cancer risk-assessment programs including genetic education, counseling, and testing became more widely available.

One of the primary aims of cancer genetic education and counseling is autonomous informed decision-making with adequate psychologic support. With those goals in mind, many cancer genetic programs are multidisciplinary and see patients

The views expressed in this article are those of the authors and do not necessarily reflect the official policy or position of the Department of the Navy, Department of Defense, or the U.S. Government.

DOI: $10.1097 /$ GIM.0b013e31812e6220 
for one or more visits before the decision to proceed with cancer predisposition testing followed by an in-person return visit for disclosure of genetic test results. ${ }^{6-8}$ The foundation for the multiple visit approach for education, counseling, and result disclosure can be traced back to a counseling protocol established for families with BRCA1 linkage. ${ }^{9}$ This model was developed using existing education, counseling, and result disclosure models for other adult-onset genetic disorders, including Huntington disease. ${ }^{10,11}$ Within that context is the premise that the physical encounter with health care providers is essential for adequate dissemination of genetic test results, as well as subsequent psychosocial assessment and support to facilitate coping with test results. Despite the 13 years that have elapsed since the first breast cancer predisposition testing protocol was published, this individualized in-person model continues to be widely used. ${ }^{12}$

The paradigm of individualized in-person encounters for genetic test result disclosure is time and provider intensive and not conducive to large-scale delivery of genetic testing services, especially in remote areas. The telephone is a reasonable option that is widely available, potentially less expensive, easy to use, and actively being studied as a means of genetic education and counseling delivery. ${ }^{13}$ Several studies on the use of the telephone to improve health behavior and health service delivery have shown that telephone-delivered interventions can reduce symptom and patient burdens and be as effective as in-person interventions in encouraging patient adherence to treatment regimens. ${ }^{14} \mathrm{~A}$ study comparing telephone breast cancer genetic education and counseling with traditional in-person education and counseling resulted in similar perceived risk and cancer worry outcomes. ${ }^{15}$ Campbell et al.'s ${ }^{16}$ study of the use of the telephone to deliver breast biopsy results found that women did as well or better hearing breast biopsy results over the phone as in-person.

In recognition that the telephone may be used for different aspects of the genetic counseling encounter, practice guidelines for the delivery of telephone genetic counseling services have been published. ${ }^{17}$ However, there are limited data available regarding outcomes associated specifically with telephone genetic test result disclosure sessions. A pilot study of telephone genetic counseling for maternal serum screening showed no differences in anxiety or knowledge between individuals receiving results in-person versus by telephone. ${ }^{18}$ There is also evidence that telephone result disclosure sessions have been used in the setting of time-sensitive BRCA1 and $B R C A 2$ test results without apparent negative consequences, although this was not a specific end point of this study. ${ }^{19}$

We report findings from a randomized study that compared the outcomes of traditional individualized in-person $B R C A 1$ and BRCA2 test result disclosure sessions with telephone disclosure sessions. We hypothesized that telephone results disclosure would result in equivalent gains in knowledge and no differences in psychologic distress measures when compared with individual in-person disclosure sessions. We also hypothesized that telephone disclosure sessions would be associated with a reduction in provider time and a reduction in patient- associated costs, and that participants would be equally satisfied with either approach to result disclosure.

\section{MATERIALS AND METHODS}

\section{Study population}

Self-selected individuals participating in a study that compared group versus individual genetics education and counseling for $B R C A 1 / B R C A 2$ testing were recruited to this result disclosure methodology study. Both studies were conducted by the National Cancer Institute and the Breast Care Center of the National Naval Medical Center and approved by the institutional review boards at both institutions. Informed consent was obtained from each subject. Findings from the primary education and counseling study are reported elsewhere but demonstrated that group education followed by brief individual counseling resulted in similar knowledge, psychologic, and satisfaction outcomes when compared with individual counseling alone with less provider time spent per patient. ${ }^{20}$

Subjects were considered eligible if they were English speaking and met the following criteria: (1) were an eligible and active participant in the primary study examining different methods of education and counseling and already consented to testing for mutations in BRCA1 and BRCA2; and (2) agreed to randomization for their test result disclosure session. Eligibility for the primary protocol included any man or woman with one of the following: (1) documented deleterious BRCA1 or $B R C A 2$ mutation in their family, (2) diagnosis of breast cancer or ductal carcinoma in situ at age $\leq 45$ years or ovarian cancer at age $\leq 50$ years; (3) men diagnosed with breast cancer at any age; and (4) personal history of breast or ovarian cancer and a family history of cancer from a three-generation pedigree resulting in a prior probability of harboring a $B R C A 1$ or $B R C A 2$ mutation that is equivalent to $10 \%$ by any peer-reviewed probability model.

A total of 136 eligible subjects were offered participation, and 111 (82\%) agreed to enroll in this study. All 25 subjects $(18 \%)$ who declined the study reported a specific preference for receiving in-person results and did not want to risk randomization to phone result disclosure. The decliners were all women but otherwise similar to the study participants in the demographic, education, and counseling methods and study variables assessed. A total of 102 of 111 subjects (92\%) completed all the portions of the study and were included in the analysis, with 50 subjects assigned to in-person results and 52 subjects assigned to phone results.

This study was designed as an equivalence study with a sample size of at least 48 subjects per arm. Forty-eight participants per study arm were determined to be sufficient to permit a change in baseline equal to 0.6 standard deviations of the difference from baseline to 1 week for either the state or trait score to have $80 \%$ power to reject the null hypothesis that the two groups were not equivalent using a two-sided alpha $=0.05$ level test. Additional subjects were enrolled to meet the minimum sample size required for analysis. 


\section{Randomization and study design}

\section{Enrollment/consent/randomization}

Subjects were recruited from the primary education and counseling protocol. Only those subjects who had completed pretest education and counseling, consented to BRCA1 and $B R C A 2$ genetic testing, and had their testing blood sample collected were eligible. Subjects were then approached by the investigator at the same appointment about the option to enroll in this aspect of the study. Those who were interested met with the investigators to review and sign a consent form that described the result disclosure process. After consent, baseline data were collected and the patient was randomized to in-person versus phone result disclosure. There were no differences between the two study arms in regard to recruitment, enrollment, or consent procedures.

\section{Pretest counseling sessions}

Participants in this study underwent pretest counseling as part of a randomized primary protocol in which 142 subjects at high risk for harboring a $B R C A$ mutation were randomized to group or individual education and counseling sessions. Group education was followed by brief individual counseling, and all sessions were conducted by genetic advanced practice nurses with training in cancer genetics. The Knowledge and Impact of Events Scale (IES) was administered at baseline, immediately after education/counseling, at 1 week, and at 3, 6, and 12 months. No difference in knowledge or IES scores were detected between those receiving individual versus group pretest education and counseling sessions. ${ }^{20}$

\section{Result sessions}

Result sessions were conducted by a physician and an advanced practice nurse in genetics, the same nurse who had been involved in the pretest counseling session when possible. Both providers were present for the entire result disclosure session including phone result sessions that used a conference-calling telephone function. Sessions were scheduled to last no more than 1 hour, and the time spent with each patient was tracked as well as who was present for the session. To minimize the differences between providers delivering results, the following format was followed for all genetic test result sessions:

- Verified the subject's continued interest in receiving genetic test results

- Provided genetic test results

- Reviewed implications of test results for the subject, including recommendations for surveillance, lifestyle modifications, and available options for follow-up care

- Assessed the response of individual and support person (if present) to the information provided

- Allowed time for questions or voicing of concerns

- Provided phone number for contact if needed

- Reviewed schedule of contacts for follow-up assessments by the genetic advanced practice nurse
- Documentation of patient response during the sessions was done immediately after the session, and a follow-up letter with results that summarized the session was provided.

\section{Long-term follow-up}

Follow-up phone calls were placed to each subject at 1 week and 3 months after the result disclosure session. These contacts were initiated to collect study outcome data; identify any unresolved psychologic or emotional issues associated with their $B R C A 1 / 2$ counseling, testing, and results; and respond to outstanding questions or concerns.

\section{Measures}

\section{Controlling variables}

Sociodemographics. At baseline as part of the study enrollment questionnaire, sex, age, marital status, race, religion, income, highest level of education, and cancer history were assessed.

Health history. As part of the primary protocol, a comprehensive health and cancer risk factor history was assessed at baseline as part of the primary enrollment questionnaire. Cancer diagnoses in all subjects were confirmed with pathology reports.

Family history. As part of the primary protocol, a bilineal three-generation family history was assessed at baseline as part of the enrollment process. Cancer diagnoses were confirmed with pathology reports only in the proband.

Genetic test results. Myriad Genetics, Inc. (Salt Lake City, Utah), a Clinical Laboratory Improvement Act-approved laboratory, performed all of the genetic testing using full-length sequencing or testing for a site-specific mutation documented in the family from a Clinical Laboratory Improvement Actapproved laboratory. In families of Ashkenazi Jewish heritage, the three-founder mutation panel was performed, and if no mutation was detected, reflex to full-length sequencing was completed. The five-mutation large rearrangement panel in BRCA1 was offered to all subjects who tested negative/uninformative or had a variant of uncertain significance.

\section{Outcome variables}

Psychologic sequelae. Once a subject consented to participate in the study (on deciding to go ahead with genetic testing and after signing the informed consent), she/he completed the Spielberger State-Trait Anxiety Inventory (STAI) (baseline), and again at 1 week and 3 months after results. The STAI was the primary tool used to evaluate the subject's anxiety related to the manner in which the subject received the test results (inperson vs. phone). ${ }^{21}$ This tool is a self-administered, 40-item questionnaire broken into two self-report scales of 20 questions, each of which takes approximately 10 minutes to complete. Each scale measures a unique anxiety concept: trait versus state. Trait anxiety is defined as a "relatively stable 
individual difference in anxiety proneness." 22 State anxiety is defined as "a transitory emotional state or condition." 22 The subject rates his or her response to each statement on a scale of 1 (not at all) to 4 (very much so). The statements relate to how one generally feels (trait) versus how one feels at a particular moment in time (state).

The STAI has test-retest reliability coefficients of 0.73 to 0.86 . Alpha coefficient values measuring internal consistency range from 0.86 to 0.92 for trait and 0.83 to 0.92 for state. Construct validity has been concluded from comparing like subjects under stressful and nonstressful situations. The STAI has been studied in varied populations, well and ill, young and elderly. The tool is written at a fifth-grade reading level.

Subjects also completed a Dupuy Psychological General Well-Being (PGWB) index. ${ }^{23}$ Individuals were asked to complete the scale 1 week after the genetic test results had been communicated (either in-person or over the phone), and again 3 months after results. The PGWB index is a subjective indicator of feelings of both well-being and distress; it has been used in the general community and takes approximately 8 to 15 minutes to complete. Developed in 1977, this tool assesses how an individual views his or her "internal personal state" versus external conditions such as income, work environment, and so forth. Six dimensions are assessed: anxiety, depression, vitality, self-control, positive well-being, and general health. The scale reflects positive and negative feelings and consists of 22 items in which the subject is asked to consider his or her feelings "during the past month." For each item there are 5-point response options representing the intensity or frequency of the experience (e.g., extremely so vs. not at all, every day vs. none of the time). A value of 0 is given for the most negative option and 5 for the most positive option. The score range for the PGWB index is 0 to 110 . Scores of 0 to 60 reflect severe distress, 61 to 72 reflect moderate distress, and 73 to 110 reflect positive well-being. Normal population survey results indicate that $71 \%$ of the adult population were in the positive well-being category. ${ }^{23}$

Test-retest reliability coefficients for the PGWB scale are 0.68 to 0.85 . The PGWB scale is reported to have high internal consistency with coefficients more than 0.94. Multiple studies have produced considerable evidence of the correlational validity of the PGWB. Average correlation of the scale and six independent depression scales was 0.69.22,24

Levels of distress associated with test results were also assessed using the IES. ${ }^{25}$ This tool is a 15 -item scale that solicits information about intrusive thoughts, avoidance, denial, and blocking of thoughts/reminders related to a specific event, in this case genetic test results. The tool has a high internal consistency of alpha 0.78 for intrusion and 0.82 for avoidance, ${ }^{25}$ and has been validated in cancer survivors. ${ }^{26}$ The majority of study participants were cancer survivors. The IES was administered at baseline and 3 , 6 , and 12 months after result disclosure.

Knowledge. Breast cancer genetics and genetic testing knowledge were assessed using a 10-item, true-false scale that was modified from the tool developed by the National Human
Genome Research Institute Cancer Genetics Studies Consortium and used in several cancer genetic studies. ${ }^{27-29}$ This tool was administered at 3, 6, and 12 months after result disclosure.

Satisfaction. A Satisfaction with Services questionnaire consisting of 10 questions was developed to assess the subject's perception of the staff's knowledge and sensitivity, and the level of satisfaction regarding the method (phone or in-person) of delivery of genetic test results. The Satisfaction with Services questionnaire was administered during the 1-week follow-up contact.

Preferred method of result disclosure. One week after the result disclosure session, all subjects were asked what method of result disclosure session they preferred, phone or in-person.

Cost analysis. Travel expense, consisting of airline ticket purchase, gas, hotel, and parking, was recorded for each subject.

Time analysis. Time to physically travel from home to the clinic was recorded for the subject and anyone who accompanied the subject to the appointment. In addition, the length of each phone and in-person session was recorded. Costs were evaluated as the value associated with the time to physically travel to and from the clinic, as well as loss of productivity (time off from work/family). ${ }^{30}$

\section{Statistical analysis}

Frequencies were calculated for each demographic and health history variable collected. These were tested for an association between the method of results (phone vs. in-person) and the following categoric variables: gender, age, race, religion, education, income, marital status, and cancer diagnosis. Analysis included the Fisher exact test for all $2 \times 2$ tables, a chi-square test for unordered tables, and a Cochran-Armitage trend test if the demographic variable was ordered. Continuous data are reported using both means and medians throughout the article because not all data were normally distributed.

In the analysis, the most important final genetic test result for each subject was established using three primary outcomes: negative/informative; positive/variant of uncertain significance and negative uninformative were combined; and positive/deleterious mutation. The final result from both $B R C A 1$ and $B R C A 2$ was combined to create a single final result variable for each subject. Stratification by affected versus unaffected with cancer and genetic test result was done to balance these factors between the in-person versus phone disclosure arms of the study.

To analyze the anxiety test data (psychologic well-being, trait anxiety, state anxiety), and the knowledge and IES data, new variables were created by subtracting the baseline value from each of the post-baseline measurements for each subject. All changes from baseline to subsequent time points were compared between the two notification groups using the Wilcoxon rank-sum test. 
Analysis of the preferred method of results used McNemar's test of agreement to test for an association between actual and preferred type of notification among those with a preference.

All $P$ values are two-tailed and have not been adjusted for multiple comparisons.

\section{RESULTS}

\section{Study population}

Subject randomization resulted in 50 subjects assigned to in-person results and 52 subjects to phone results. Table 1 provides an overview of the study population characteristics. Participants ranged in age from 25 to 68 years, most were female $(93 \%)$ and white $(93 \%)$, and $72 \%$ were affected with breast cancer. Most participants were married (72\%), 65\% reported a college degree or higher level of education, and $74 \%$ had an income of more than $\$ 50,000$ per year. Although there are some trends toward differences in race, religion, and marital status between the two arms, in view of the number of parameters evaluated, none of the differences between the phone and in-person results groups for the demographic variables assessed could be considered statistically significant.

\section{Trait anxiety}

Outcomes from the Spielberger tool measurement of trait anxiety are shown in Table 2. Trait anxiety indicates how participants generally feel or the individual difference in anxiety proneness. The mean and median state anxiety scores at baseline, at 1 week after results, and at 3 months were measured. Results can vary from a range of low anxiety (20) to high anxiety (80). Median trait anxiety scores were 26 to 31 throughout, representing an overall low trait anxiety throughout with no significant difference in anxiety between participants receiving phone or in-person results.

\section{State anxiety}

Outcomes from the Spielberger tool measurement of state anxiety are shown in Table 2 . The mean and median state anxiety scores at baseline, 1 week after results, and at 3 months indicated how study participants felt at that particular point in time. Results can vary from a range of low anxiety (20) to high anxiety (80). Participants experienced median state anxiety scores of 24 to 27 throughout. The phone notification group had a median decline of 1 point in the state anxiety scale from baseline to 1 week, whereas the in-person group had a median increase of 3 points, which was a marginally statistically significant difference between the two arms $(P=0.025)$ in view of the multiple comparisons undertaken. This difference was not present at the 3-month follow-up.

\section{General well-being}

Table 2 provides the outcome measurement scores for general well-being and distress, which can range from 0 (most negative) to 110 (most positive). In general, individuals in this study reported high levels of general well-being that did not change much over the follow-up period. Participants had a positive well-being
Table 1

Demographics of study population

\begin{tabular}{|c|c|c|c|}
\hline Demographics & $\begin{array}{l}\text { In-person } \\
(N=50)\end{array}$ & $\begin{array}{c}\text { Phone } \\
(N=52)\end{array}$ & $\begin{array}{c}P \text { value } \\
\text { (Two-tailed) }\end{array}$ \\
\hline Sex & & & 0.71 \\
\hline Male & 4 & 3 & \\
\hline Female & 46 & 49 & \\
\hline Age (y) & & & 0.91 \\
\hline Median (range) & $45(25-68)$ & $45(20-70)$ & \\
\hline Race & & & 0.042 \\
\hline White & 46 & 49 & \\
\hline Asian & 0 & 1 & \\
\hline Black & 4 & 0 & \\
\hline Hispanic & 0 & 2 & \\
\hline Religion & & & 0.031 \\
\hline Catholic & 9 & 19 & \\
\hline Jewish & 7 & 12 & \\
\hline Protestant & 25 & 16 & \\
\hline Other & 9 & 4 & \\
\hline Missing & 0 & 1 & \\
\hline Education & & & 0.84 \\
\hline High school \pm some college & 17 & 19 & \\
\hline College degree or higher & 33 & 33 & \\
\hline Income & & & 0.37 \\
\hline$<\$ 25,000$ & 5 & 2 & \\
\hline$\$ 25,000-\$ 50,000$ & 10 & 9 & \\
\hline$\$ 50,000-\$ 75,000$ & 9 & 11 & \\
\hline$>\$ 75,000$ & 26 & 29 & \\
\hline Missing & 0 & 1 & \\
\hline Marital status & & & $0.054^{a}$ \\
\hline Single & 8 & 2 & \\
\hline Married & 31 & 42 & \\
\hline Divorced & 6 & 3 & \\
\hline Separated & 1 & 0 & \\
\hline Significant other & 0 & 1 & \\
\hline Widowed & 3 & 1 & \\
\hline Missing & 1 & 3 & \\
\hline Cancer history & & & 0.89 \\
\hline Unaffected & 9 & 12 & \\
\hline Breast cancer & 37 & 36 & \\
\hline Ovarian cancer & 4 & 4 & \\
\hline
\end{tabular}

${ }^{a} P=0.019$ for comparison of married versus nonmarried subjects between arms.

throughout with no significant difference noted according to result methodology. Furthermore, no statistical difference was detected on the basis of result disclosure method and pretest counseling method, individual versus group. 
Table 2

Psychologic sequelae

\begin{tabular}{|c|c|c|c|}
\hline Measurement & $\begin{array}{c}\text { In-person } \\
\text { mean (SD), median (range) }\end{array}$ & $\begin{array}{c}\text { Phone } \\
\text { mean }(\mathrm{SD}), \text { median (range) }\end{array}$ & $\begin{array}{l}P \text { value for comparison } \\
\text { between groups for } \\
\text { change from baseline }\end{array}$ \\
\hline \multicolumn{4}{|c|}{ Trait anxiety (range $20=$ low to $80=$ high $)$} \\
\hline Baseline & $30.27(7.48) 28(20-53)$ & $30.54(6.81) 29(20-48)$ & - \\
\hline $1 \mathrm{wk}$ & $30.46(7.44) 31(20-52)$ & $30.69(9.09) 29(20-61)$ & 0.17 \\
\hline $3 \mathrm{mo}$ & $28.77(7.60) 26(20-48)$ & $28.67(7.28) 26(20-54)$ & 0.83 \\
\hline \multicolumn{4}{|c|}{ State anxiety (range $20=$ low to $80=$ high $)$} \\
\hline Baseline & $27.8(7.75) 26(20-48)$ & $30.12(10.84) 27(20-68)$ & - \\
\hline $1 \mathrm{wk}$ & $29.7(9.83) 26(20-59)$ & $28.88(9.77) 27(20-68)$ & $0.025^{a}$ \\
\hline $3 \mathrm{mo}$ & $27.81(9.26) 24(20-58)$ & $29.41(9.19) 24(20-58)$ & 0.58 \\
\hline \multicolumn{4}{|c|}{ General well-being (range $0=$ most negative to $110=$ most positive) } \\
\hline Baseline & $83.06(14.87) 87(43-105)$ & $83.17(14.73) 86(27-108)$ & - \\
\hline $1 \mathrm{wk}$ & $83.46(15.17) 85.5(45-108)$ & 82.98 (15.61) $85(33-109)$ & 0.49 \\
\hline $3 \mathrm{mo}$ & $84.57(14.16) 88(51-106)$ & $85.33(12.79) 89(48-110)$ & 0.34 \\
\hline \multicolumn{4}{|c|}{ IES scores $($ low $<8.5$, medium $=8.5-18$, high $>18)$} \\
\hline Baseline & $8.14(8.44) 6.00(0-29)$ & $11.96(14.18) 8.00(0-63)$ & N/A \\
\hline $3 \mathrm{mo}$ & $6.52(10.65) 1.00(0-40)$ & $7.34(9.87) 5.00(0-50)$ & 0.46 \\
\hline $6 \mathrm{mo}$ & $8.04(12.54) 0.50(0-46)$ & $7.38(9.46) 2.00(0-31)$ & 0.42 \\
\hline $12 \mathrm{mo}$ & $6.10(8.60) 1.00(0-34)$ & $5.83(8.98) 1.00(0-35)$ & 0.83 \\
\hline
\end{tabular}

SD, standard deviation; N/A, not applicable; IES, Impact of Events Scale.

${ }^{a}$ Statistically significant difference between groups with respect to change from baseline.

\section{Level of distress}

Psychologic distress as measured by the IES indicated a low level of distress throughout the 1-year measurement period. The mean and median IES scores at baseline and 3, 6, and 12 months are shown in Table 2. There was no significant difference in the changes in IES scores between those receiving phone or in-person genetic test results.

IES scores of the 26 subjects who declined randomization were not significantly different from those of the study participants.

\section{Genetic test results}

Twenty seven (26\%) deleterious mutations, 19 mutations in $B R C A 1$, and 8 mutations in BRCA2 were detected. Ten subjects $(10 \%)$ were tested for a known mutation in the family and found to have negative results. Seventeen subjects (17\%) were found to have a variant of uncertain significance in either the $B R C A 1$ and/or BRCA2 gene. No BRCA mutations were detected in the remaining 49 subjects (48\%). Deleterious mutation carriers were equally distributed between the two arms of the study.

\section{Knowledge}

Outcomes from the scale scores in each group are depicted in Table 3. There was a gain in knowledge when compared with baseline that persisted throughout the 12-month follow-up pe- riod. The median difference in knowledge scores from baseline to post-education and counseling persisted for each follow-up measurement ( 3,6 , and 12 months). There was no difference in the change in knowledge scores between those receiving phone versus in-person test results.

General satisfaction. Participants indicated a high level of satisfaction with the process of receiving genetic test results. On a scale of $0=$ low satisfaction to $32=$ high satisfaction, general satisfaction scores ranged from 23 to 32 . There was no significant difference with satisfaction with services between those who received phone, mean 30.6 (range 23-32), and those who received in-person results, mean 29.8 (range 23-32).

Method of result disclosure satisfaction. Participants were asked to indicate satisfaction with their actual method of result provision on a scale of 1 to 4 , with $1=$ very dissatisfied, $2=$ dissatisfied, $3=$ satisfied, and $4=$ very satisfied. Satisfaction with phone results, mean 3.98 (range 3-4), was slightly higher than for in-person results, mean 3.56 (range 2-4). At the 1-week follow-up, participants were asked what method of result disclosure they preferred. Of those who received an inperson result session, 28 (57\%) reported they preferred inperson results, $20(41 \%)$ indicated they preferred phone results, and $1(2 \%)$ preferred either method. In contrast, 46 $(90 \%)$ who had a phone result indicated they preferred phone 
Table 3

Knowledge scores $(0=$ no questions correct, $10=$ perfect score $)$

\begin{tabular}{lccc}
\hline Measurement & $\begin{array}{c}\text { In-person results scores } \\
\text { median (range) mean (SD) }\end{array}$ & $\begin{array}{c}\text { Phone results scores } \\
\text { median (range) mean (SD) }\end{array}$ & $\begin{array}{c}\text { P value for comparison between } \\
\text { groups for change from baseline }\end{array}$ \\
\hline Baseline & $9.0(3-10)$ & $8.0(1-10)$ & N/A \\
Post-education and counseling & $8.12(1.7)$ & $7.83(2.08)$ & $10.0(8-10)$ \\
& $10.0(7-10)$ & $9.79(0.46)$ & $10.0(7-10)$ \\
3 mo & $9.68(0.71)$ & $9.40(0.80)$ & $10.0(7-10)$ \\
6 mo & $10.0(7-10)$ & $9.51(0.79)$ & 0.62 \\
12 mo & $9.36(0.82)$ & $10.0(7-10)$ & $9.38(0.91)$ \\
\hline
\end{tabular}

SD, standard deviation; N/A, not applicable.

results, $2(4 \%)$ preferred in-person results, and $3(6 \%)$ preferred either method. The data show that the majority of participants in both groups (74/96 with preference; 77\%) were satisfied with the method of result disclosure to which they were randomized. However, among those with a stated preference who did not receive their preferred method ( $n=22$ overall), there was a statistically significant tendency to prefer phone results ( 20 vs. 2 ; $P<0.0001$ by McNemar test for agreement in paired categorical data). None of the participants who received phone results requested an in-person follow-up session.

\section{Costs}

Greater costs were associated with travel and time needed for those coming to the clinic for in-person test results (Table 4). Some subjects were military personnel stationed in foreign countries, and their travel time, cost, and required time off was greatest. Individuals who had support persons travel with them to the clinic had additional costs in terms of their time off, child care, and pet care. For phone results, phone charges were not measured because the calls were made from a government facility with covered access to long-distance calls. A few persons receiving phone results reflected minimal travel costs, with some support persons traveling to them or joining in the call for the result session. Because this study provided results as part of a research study, no charges were designated for the professional time for the session.

\section{Time}

Time spent by the professional was comparable for both in-person (mean 44 minutes) and phone results (mean 39 minutes). No patients required more than the 1 hour allocated for the result disclosure appointment.

\section{DISCUSSION}

Testing for mutations in BRCA1 and BRCA2 has already entered a direct-to-consumer marketing era, raising the possibility of an increase in demand for testing. Given the limited number of cancer genetic providers available to deliver these services, novel mechanisms for service delivery are needed. These data demonstrate that phone genetic test result disclosure sessions are equivalent to in-person disclosure sessions in terms of anxiety experienced, general well-being, and satisfaction with services. When stratified by genetic test results (positive, negative for known mutation in family, negative/uninformative, variant of uncertain significance), there was no difference between phone or in-person result groups in trait anxiety, state anxiety, or general well-being.

To clarify the significance of our knowledge and affective assessments, anchoring our findings to those in comparable populations is valuable. ${ }^{31} \mathrm{We}$ compared our findings with those of similar studies using populations of individuals undergoing genetic counseling for familial cancer. ${ }^{32}$ Those studies found baseline mean state anxiety scores of 11.5 to 38.7 and

Table 4

Costs

\begin{tabular}{|c|c|c|c|c|}
\hline Measurement & Round trip travel time mean/range & Round trip miles mean/range & Travel cost mean/range & Time off range \\
\hline In-person & $6 \mathrm{~h}(0.25-65)$ & 236 miles $(3-1224)$ & $\$ 120(\$ 5-550)$ & $2 \mathrm{~h}$ to $1 \mathrm{wk}$ \\
\hline Phone & None $(0-0.75)$ & None (0-45) & None & $1-4 \mathrm{~h}$ \\
\hline
\end{tabular}


follow-up mean scores of 10.3 to $37.3 .{ }^{32}$ Those studies found IES mean scores at baseline ranged from 13.1 to 15.1 and follow-up mean scores ranged from 10.3 to $13.8 .{ }^{32}$ Scores for similar measures in this study were comparable and demonstrate similar low levels of anxiety and distress throughout.

Among those who expressed a preference and did not prefer the method to which they were assigned, a statistically significant preference for phone results was identified. Our experience has revealed that for those who consider using phone results in their practice, preparation for the logistics of a phone disclosure session is necessary. Not all patients recognize the need for the conversation to take place in a private location with minimal distractions or allocate sufficient time for the session. Some settings are less conducive to thoughtful interaction, discussion, and privacy, so encouraging the patient to consider these issues when scheduling a phone appointment is helpful. In addition, cellular phones allow one to make and receive calls in a wide variety of public places, so individuals need to be encouraged to have this conversation in a private place without any distraction.

Provider skills and comfort during the interactions (whether phone or in-person) may have affected study outcomes. Provider perceptions were not assessed as part of this study, although some providers expressed concern because they could not rely on the usual physical or emotional cues during telephone notification. Specific training and practice with provision of phone results may be needed for some providers to enhance sensitivity to the pace of the session and attention to tone of voice and enhance telephone assessment skills. Videoconference technology could be considered to aid in the assessment of the patient during the session, although this would be limited to those with access to that technology. ${ }^{33}$

Eighteen percent of potential subjects for this study declined to participate because they preferred to obtain results in-person. Assessing an individual's preference or need for in-person genetic test result disclosure is essential. Consideration for an in-person encounter might also be warranted for those requiring complex result notification, expressing high anxiety, or indicating unclear communication of difficult concepts. ${ }^{17}$ For all others, phone results would be a reasonable alternative.

Telephone care is frequently not billed for or reimbursed by most health plans, and therefore a result disclosure session delivered by telephone may not be a billable service. ${ }^{34}$ Review of billing practices, including whether to bill for each session independently or as a bundled service, may facilitate reimbursement for costs of these services. In practice, balancing patient preference and cost savings with the potential loss of revenue for resource use is a consideration.

Breast cancer predisposition genetic testing has implications for individuals and their family members. One significant advantage of receiving phone test results was the opportunity for family members from across the country (some of whom might otherwise have been unable to participate) to partake in the disclosure session. Not only did those individuals avoid the stress and expense of travel to the clinic, but the ability for direct provider to family member discussion may have been of benefit.

Participants in this study were originally entered in a randomized study that compared group versus individual genetic education and counseling for $B R C A 1 / 2$ testing. On acceptance into that study, they were then offered further randomization for test result delivery method. The primary study found no significant difference in knowledge, psychologic, and satisfaction outcomes when comparing traditional individual counseling with group education followed by brief individual counseling. ${ }^{20}$ The pretest education method may have influenced the findings in this aspect of the study. However, both pretest education and counseling methods were found to be equivalent over the 12-month follow-up period, reducing the likelihood that participation in the primary study affected the findings in this aspect of the study. Despite the primary studies equivalent findings, this study was conducted on a research cohort drawn from another study with travel, education, counseling, and testing offered at no cost, which is a major limitation potentially influencing study outcomes, including participant satisfaction. Therefore, the study should be replicated in a population not already participating in a genetic testing protocol.

This study was not powered for a subgroup analysis that looked specifically at delivery of deleterious mutation results, or other result category, compared with any other test results within each study arm. Stratification to each of the two result methods, including affected versus unaffected with cancer and genetic test result type, was included to balance these factors between study arms. Participants were primarily white, highly educated, and of a higher socioeconomic bracket than is representative of the general population. In addition, the majority of participants were female and several came from a military institution. Generalizability of these results may be limited by these factors, and future research in a more diversified population that includes more men and individuals found to harbor a deleterious mutation is needed.

\section{CONCLUSION}

The use of telephone genetic test result disclosure sessions provides an alternative to standard in-person result disclosure without negatively affecting satisfaction, knowledge, or psychologic distress. Result disclosure sessions should provide similar content and support regardless of methodology. Sensitivity to psychologic well-being, ability to determine preference for disclosure, and preference for in-person result disclosure should be considered by the health care provider. These data suggest that for patients willing and interested in phone results, $B R C A 1 / 2$ telephone result disclosure is a reasonable alternative to traditional in-person genetic test disclosure for the majority of patients. Further research in a representative population will be needed to confirm these findings. 


\section{ACKNOWLEDGMENTS}

This research was supported [in part] by the Intramural Research Program of the National Institutes of Health, National Cancer Institute, Center for Cancer Research.

\section{References}

1. Cancer facts and figures, 2006. Atlanta, GA: American Cancer Society, 2006.

2. Claus EB, Schildkraut JM, Thompson WD, Risch NJ. The genetic attributable risk of breast and ovarian cancer. Cancer 1996;77:2318-2324.

3. Miki Y, Swensen J, Shattuck-Eidens D, et al. A strong candidate for the breast and ovarian cancer susceptibility gene BRCA1. Science 1994;266:66-71.

4. Tavtigian SV, Simard J, Rommens J, et al. The complete BRCA2 gene and mutations in chromosome 13q-linked kindreds. Nat Genet 1996;12:333-337.

5. Wooster R, Bignell G, Lancaster J, et al. Identification of the breast cancer susceptibility gene BRCA2. Nature 1995;378:789-792.

6. Dimond E. Establishing a cancer genetics clinic. In: Genetics in oncology practice: cancer risk assessment. Tranin A, Masny A, Jenkins J, editors. Pittsburgh: Oncology Nursing Society, 2003:225-241.

7. van Oostrom I, Tibben A. A counseling model for BRCA1/2 genetic susceptibility testing. Hereditary Cancer in Clinical Practice 2004;2:19-23.

8. Calzone KA, Stopfer J, Blackwood A, et al. Establishing a cancer risk evaluation program. Cancer Pract 1997;5:228-233.

9. Biesecker BB, Boehnke M, Calzone K, et al. Genetic counseling for families with inherited susceptibility to breast and ovarian cancer. J Am Med Assoc 1993;269: 1970-1974.

10. America THsDSo: Guidelines for predictive testing for Huntington's disease. New York: Huntington's Disease Society of America, Inc., 1989.

11. Chorea IHA at WFoNRGoHs: guidelines for the molecular genetics predictive test in Huntington's disease. J Med Genet 1994;31:555-559.

12. Thompson JA, Wiesner GL, Sellers TA, et al. Genetic services for familial cancer patients: a survey of National Cancer Institute cancer centers. J Natl Cancer Inst 1995;87:1446-1455.

13. Wang V. Commentary: what is and is not telephone counseling. J Genet Couns 2000;9:73-82.

14. McBride CRB. Using the telephone to improve health behavior and health service delivery. Patient Educ Couns 1999;37:3-18.

15. Helmes AW, Culver JO, Bowen DJ. Results of a randomized study of telephone versus in-person breast cancer risk counseling. Patient Educ Couns 2006;64:96-103.

16. Campbell L, Watkins RM, Teasdale C. Communicating the result of breast biopsy by telephone or in person. Br J Surg 1997;84:1381.

17. Ormond KE, Haun J, Cook L, Duquette D, Ludowese C, Matthews AL. Recommendations for telephone counseling. J Genet Couns 2000;9:63-71.
18. Sangha KK, Dircks A, Langlois S. Assessment of the effectiveness of genetic counseling by telephone compared to a clinic visit. J Genet Couns 2003;12: 171-184.

19. Schwartz MD, Lerman C, Brogan B, et al. Impact of BRCA1/BRCA2 counseling and testing on newly diagnosed breast cancer patients. J Clin Oncol 2004;22: 1823-1829.

20. Calzone KA, Prindiville SA, Jourkiv O, et al. Randomized comparison of group versus individual genetic education and counseling for familial breast and/or ovarian cancer. J Clin Oncol 2005;23:3455-3464.

21. Spielberger C. State-Trait Anxiety Inventory for adults. Menlo Park, CA: Mind Garden, 1977.

22. Frank-Stromberg M, Olsen S. Instruments for clinical health-care research. Boston, MA: Jones \& Bartlett Publishers, 1997.

23. Dupuy H. The Psychological General Well-Being (PGWB) Index. Shelton, CT: Le Jacq Publishing, 1984.

24. McDowell I, Newell C. Measuring health (ed 2nd). New York: Oxford University Press, 1996.

25. Horowitz M, Wilner N, Alvarez W. Impact of event scale: a measure of subjective stress. Psychosom Med 1979;41:209-218.

26. Zilberg NJ, Weiss DS, Horowitz MJ. Impact of event scale: a cross-validation study and some empirical evidence supporting a conceptual model of stress response syndromes. J Consult Clin Psychol 1982;50:407-414.

27. Lerman C, Biesecker B, Benkendorf JL, et al. Controlled trial of pretest education approaches to enhance informed decision-making for BRCA1 gene testing. J Nat Cancer Inst 1997;89:148-157.

28. Bluman LG, Rimer BK, Berry DA, et al. Attitudes, knowledge, and risk perceptions of women with breast and/or ovarian cancer considering testing for BRCA1 and BRCA2. J Clin Oncol 1999;17:1040-1046.

29. Hughes C, Gomez-Caminero A, Benkendorf J, et al. Ethnic differences in knowledge and attitudes about BRCAl testing in women at increased risk. Patient Educ Couns 1997;32:51-62.

30. Stone P. Methods for conducting and reporting cost-effectiveness analysis in nursing. Image 1998;30:229-234.

31. Coyne JC, Kruus L, Racioppo M, Calzone KA, Armstrong K. What do ratings of cancer-specific distress mean among women at high risk of breast and ovarian cancer? Am J Med Genet 2003;116A:222-228.

32. Braithwaite D, Emery J, Walter F, Prevost T, Sutton S. Psychological impact of genetic counseling for familial cancer: a systematic review and meta-analysis. J Nat Cancer Inst 2004;96:122-133.

33. Craig J, Patterson V. Introduction to the practice of telemedicine. J Telemed Telecare 2005;11:3-9.

34. Melzer S, Poole S. Reimbursement for telephone care. Pediatrics 2002;109: 290-293. 